December 1997

\title{
Screening Current Representation of Quantum Superalgebras
}

\author{
Jørgen Rasmussen円 \\ Laboratoire de Mathémathiques et Physique Théorique, \\ Université de Tours, F-37200 Tours, France
}

\begin{abstract}
In this letter a screening current or contour representation is given of certain quantum superalgebras. The Gomez-Sierra construction of quantum groups in conformal field theory is generalized to cover superalgebras and illustrated using recent results on screening currents in affine current superalgebra.
\end{abstract}

PACS: $11.25 . \mathrm{Hf}$

Keywords: Affine current superalgebra; Conformal field theory; Quantum group

\footnotetext{
${ }^{1}$ e-mail address: jorgen@celfi.phys.univ-tours.fr
} 


\section{Introduction}

Ever since their appearances [1, 2] quantum groups have played an increasingly significant role in both mathematics and theoretical physics. An important reason is due to their property of bridging various areas of mathematical physics. Also quantum supergroups have attracted a great deal of attention, see e.g. [3] and the review [4].

In the work [5] by Gomez and Sierra a particular construction of $q$-deformed universal enveloping algebras was introduced in the context of minimal models in conformal field theory. It is based on vertex operators screened by integrated screening currents, and by use of contour manipulations the quantum group structure is revealed. Their result allowed for an immediate generalization to affine current algebras [6], and in [7] a general scheme for obtaining the Gomez-Sierra construction is discussed. However, the generality is limited to purely bosonic cases. The object of the present letter is to generalize their scheme to include the case of superalgebras. The particular example of affine current superalgebras [8] is then discussed.

The remaining part of this letter is organized as follows. In Section 2 we discuss in a general setting the Gomez-Sierra construction in the case of superalgebras, closely following the original works on the purely bosonic cases [5, 6, 7]. In Section 3 we then illustrate the generalization by considering affine current superalgebras using recent results on those obtained by the present author in [8]. Section 4 contains some concluding remarks, in particular some speculations on a possible generalization by including also screening currents of the second kind [9, 10, 11, 12]. The construction obtained in affine current algebra [6] and the one in this letter in affine current superalgebra are based only on screening currents of the first kind.

\section{Gomez-Sierra Construction for Superalgebras}

The discussion here is in the framework of conformal field theory. It is assumed that the conformal field theory under consideration admits a Coulomb gas or Feigin-Fuks representation. This ensures the existence of a set of screening currents where a screening current has the property that the singular part of the operator product expansion with a chiral algebra generator is a total derivative. In particular, the screening currents are conformally primary of weight 1 . These properties ensure that integrated screening currents (screening charges) may be inserted into correlators in the conformal field theory without altering the chiral (including the conformal) Ward identities. This in turn makes them very useful in construction of correlators, see e.g. [13, 14, 15, 10.

Alternatively one may use the screening currents in representing certain quantum algebras [5, 6, 7]. The contours used in the latter case are different from and simpler than the ones needed in the former case. In this section we shall generalize the latter construction of purely bosonic quantum groups to quantum supergroups.

Let $\left\{S_{j}(w)\right\}_{j \in \mathcal{S}}$ be a set of screening currents and let $V_{\lambda}(z)$ denote a generic vertex operator of weight $\lambda$. The parities of the fields are defined by

$$
p\left(V_{\lambda}\right)=0 \quad, \quad p\left(S_{j}\right)= \begin{cases}1 & , j \in \tau \\ 0 & , j \in \mathcal{S} \backslash \tau\end{cases}
$$


where $\tau$ is a subset of $\mathcal{S}$. The construction of the quantum superalgebra based on the following defining braiding properties

$$
\begin{aligned}
V_{\lambda}(z) V_{\lambda^{\prime}}(w) & =e^{i \pi \Omega_{\lambda \lambda^{\prime}}} V_{\lambda^{\prime}}(w) V_{\lambda}(z) \\
S_{j}(z) V_{\lambda}(w) & =e^{i \pi \Omega_{j \lambda}} V_{\lambda}(w) S_{j}(z) \\
S_{i}(z) S_{j}(w) & =e^{i \pi \hat{\Omega}_{i j}} S_{j}(w) S_{i}(z) \\
& =e^{i \pi\left(\Omega_{i j}+p\left(S_{i}\right) p\left(S_{j}\right)\right)} S_{j}(w) S_{i}(z)
\end{aligned}
$$

is equivalent to the one used in the bosonic case [7]. Thus we will follow that but, however, use a slightly different notation. The matrices $\Omega_{i j}$ and $\Omega_{\lambda \lambda^{\prime}}$ are assumed symmetric.

First one introduces for every $z$ a vector space $\mathcal{V}_{\lambda, z}$, spanned by screened vertex operators $U_{\lambda, I}(z)$

$$
U_{\lambda, I}(z)=\int_{C_{1}} d w_{1} S_{i_{1}}\left(w_{1}\right) \ldots \int_{C_{|I|}} d w_{|I|} S_{i_{|I|}}\left(w_{|I|}\right) V_{\lambda}(z)
$$

where $I=\left\{i_{1}, \ldots, i_{|I|}\right\}$ is an ordered set of labels of screening currents. The contours all start at infinity, encircle counter-clockwise the branch point $z$ once, and return to infinity. They do not intersect, nor do they cross the branch cut from $z$ to infinity. The ordering of the contours is chosen such that $C_{i}$ lies inside $C_{j}$ for $i>j$. Such contours will be denoted standard contours around $z$. The vertex operator itself belongs to $\mathcal{V}_{\lambda, z}$, namely $V_{\lambda}(z)=U_{\lambda, \emptyset}(z)$. It is called the highest weight vector, distinguished by the absence of contour structure.

For every screening current $S_{j}$ one defines a quartet of operators $F_{j}, K_{j}^{ \pm 1}$ and $E_{j}$. The former is defined as the contour creating operator

$$
F_{j}\left(U_{\lambda, I}(z)\right)=\int_{C} d w S_{j}(w) U_{\lambda, I}(z)
$$

where $C$ is a standard contour around $z$ and encloses the ones in $U_{\lambda, I}(z)$. By this definition $F_{j}$ inherits the parity of $S_{j}$. Now, choosing the branch cuts inherent in $\mathcal{V}_{\lambda_{1}, z_{1}}$ and $\mathcal{V}_{\lambda_{2}, z_{2}}$ non-intersecting, we may define the co-multiplication $\Delta\left(F_{j}\right)$ on $\mathcal{V}_{\lambda_{1}, z_{1}} \otimes \mathcal{V}_{\lambda_{2}, z_{2}}$ by

$$
\begin{aligned}
\Delta\left(F_{j}\right)\left(U_{\lambda_{1}, I_{1}}\left(z_{1}\right) \otimes U_{\lambda_{2}, I_{2}}\left(z_{2}\right)\right) & =\int_{C} d w S_{j}(w) U_{\lambda_{1}, I_{1}}\left(z_{1}\right) U_{\lambda_{2}, I_{2}}\left(z_{2}\right) \\
& =F_{j}\left(U_{\lambda_{1}, I_{1}}\left(z_{1}\right)\right) U_{\lambda_{2}, I_{2}}\left(z_{2}\right) \\
& +e^{i \pi\left(\sum_{i \in I_{1}} \hat{\Omega}_{j i}+\Omega_{j \lambda_{1}}\right)} U_{\lambda_{1}, I_{1}}\left(z_{1}\right) F_{j}\left(U_{\lambda_{2}, I_{2}}\left(z_{2}\right)\right)
\end{aligned}
$$

The contour $C$ is of the standard type wrt the pair $z_{1}$ and $z_{2}$. By an obvious contour deformation it may be written as a sum $C=C_{1}+C_{2}$ where $C_{1}\left(C_{2}\right)$ is a standard contour around $z_{1}\left(z_{2}\right)$. Thereby one has obtained

$$
\Delta\left(F_{j}\right)=F_{j} \otimes 1+K_{j}^{-1} \otimes F_{j}
$$

where $K_{j}$ (and $K_{j}^{-1}$ ) is defined by

$$
K_{j}\left(U_{\lambda, I}(z)\right)=e^{-i \pi\left(\sum_{i \in I} \Omega_{j i}+\Omega_{j \lambda}\right)} U_{\lambda, I}(z)
$$


Note that

$$
\left(A_{1} \otimes A_{2}\right)\left(U_{1} \otimes U_{2}\right)=(-1)^{p\left(A_{2}\right) p\left(U_{1}\right)} A_{1}\left(U_{1}\right) \otimes A_{2}\left(U_{2}\right)
$$

The co-multiplication of $K_{j}^{ \pm 1}$ is trivial

$$
\Delta\left(K_{j}^{ \pm 1}\right)=K_{j}^{ \pm 1} \otimes K_{j}^{ \pm 1}
$$
by

The raising operator $E_{j}$ is related to the operator $\hat{E}_{j}$ (see below (17)) defined implicitly

$$
\begin{aligned}
L_{-1} U_{\lambda, I}(z) & =\left[L_{-1}, \prod_{i_{l} \in I} \int_{C_{l}} d w_{l} S_{i_{l}}\left(w_{l}\right)\right] V_{\lambda}(z)+\prod_{i_{l} \in I} \int_{C_{l}} d w_{l} S_{i_{l}}\left(w_{l}\right) L_{-1} V_{\lambda}(z) \\
& =-\sum_{j \in \mathcal{S}}\left(q_{j}-q_{j}^{-1}\right) S_{j}(\infty) \hat{E}_{j} U_{\lambda, I}(z)+\prod_{i \in I} F_{i} L_{-1} V_{\lambda}(z)
\end{aligned}
$$

The last term is a screened descendant of $V_{\lambda}$. At this point the factor $\left(q_{j}-q_{j}^{-1}\right)$ is merely a normalization constant. In the application in Section 3 on affine current superalgebras we shall see that choosing $q_{j} \neq 1$ appropriately (and related to $e^{i \pi \Omega_{j j}}$ ), we obtain a $q$-deformed Lie superalgebra. From

$$
\begin{aligned}
{\left[L_{-1}, \prod_{i_{l} \in I} \int_{C_{l}} d w_{l} S_{i_{l}}\left(w_{l}\right)\right] V_{\lambda}(z) } & =-\sum_{i_{l} \in I}\left(q_{i_{l}}-q_{i_{l}}^{-1}\right) S_{i_{l}}(\infty) \frac{1-e^{2 \pi i\left(\sum_{i^{\prime}>i_{l}} \hat{\Omega}_{i_{l^{i^{\prime}}}+\Omega_{i_{l} \lambda}}\right)}}{q_{i_{l}}-q_{i_{l}}^{-1}} \\
& \cdot e^{i \pi \sum_{i^{\prime}<i_{l}} \hat{\Omega}_{i_{l^{\prime}} i^{\prime}}} \prod_{i_{l^{\prime}} \in I \backslash\left\{i_{l}\right\}} \int_{C_{l^{\prime}}} d w_{l^{\prime}} S_{i_{l^{\prime}}}\left(w_{l^{\prime}}\right) V_{\lambda}(z)
\end{aligned}
$$

we obtain

$$
\hat{E}_{j} U_{\lambda, I}=\sum_{i_{l} \in I, i_{l} \sim j} \frac{1-e^{2 \pi i\left(\sum_{i^{\prime}>i_{l}} \Omega_{j i^{\prime}}+\Omega_{j \lambda}\right)}}{q_{j}-q_{j}^{-1}} e^{i \pi \sum_{i^{\prime}<i_{l}} \hat{\Omega}_{j i^{\prime}}} U_{\lambda, I \backslash\left\{i_{l}\right\}}
$$

Here we have used that $U_{\lambda, I \backslash\{i\}}=0$ for $i \notin I$. The restriction in the summation means $i_{l} \sim j$ if $S_{i_{l}}=S_{j}$. (12) is a simple generalization of the analogous result for purely bosonic generators [10]. Note that just like $F_{j}, \hat{E}_{j}$ inherits the parity of $S_{j}$. In the special case of only one type of screening current, (12) reduces to $\left(U_{\lambda ;|I|}=U_{\lambda, I}\right)$

$$
\hat{E}_{j} U_{\lambda ;|I|}=\frac{1-e^{2 \pi i \Omega_{j \lambda}} e^{i \pi(|I|-1) \hat{\Omega}_{j j}}}{q_{j}-q_{j}^{-1}}[|I|]_{e^{i \pi \hat{\Omega}_{j j}}} U_{\lambda ;|I|-1}
$$

This is in agreement with [0] in the purely bosonic case. The $q$-number is introduced as

$$
[a]_{q}=\frac{1-q^{a}}{1-q}
$$

The co-multiplication of $\hat{E}_{j}$ is defined implicitly using the trivial co-multiplication of $L_{-1}\left(\right.$ which is $\left.\Delta\left(L_{-1}\right)=L_{-1} \otimes 1+1 \otimes L_{-1}\right)$

$$
\begin{aligned}
& \Delta\left(L_{-1}\right)\left(U_{\lambda_{1}, I_{1}}\left(z_{1}\right) \otimes U_{\lambda_{2}, I_{2}}\left(z_{2}\right)\right) \\
= & \prod_{i \in I_{1}} F_{i} L_{-1} V_{\lambda_{1}}\left(z_{1}\right) \otimes U_{\lambda_{2}, I_{2}}\left(z_{2}\right)+U_{\lambda_{1}, I_{1}}\left(z_{1}\right) \otimes \prod_{i \in I_{2}} F_{i} L_{-1} V_{\lambda_{2}}\left(z_{2}\right) \\
- & \sum_{j \in \mathcal{S}}\left(q_{j}-q_{j}^{-1}\right) S_{j}(\infty) \Delta\left(\hat{E}_{j}\right)\left(U_{\lambda_{1}, I_{1}}\left(z_{1}\right) \otimes U_{\lambda_{2}, I_{2}}\left(z_{2}\right)\right)
\end{aligned}
$$


and is found to be

$$
\Delta\left(\hat{E}_{j}\right)=\hat{E}_{j} \otimes 1+K_{j}^{-1} \otimes \hat{E}_{j}
$$

After having redefined the raising operator as

$$
E_{j}=K_{j} \hat{E}_{j}
$$

the co-multiplication reads

$$
\Delta\left(E_{j}\right)=E_{j} \otimes K_{j}+1 \otimes E_{j}
$$

With the following definitions of an anti-pode $\gamma$

$$
\gamma\left(E_{j}\right)=-E_{j} K_{j}^{-1} \quad, \quad \gamma\left(K_{j}^{ \pm 1}\right)=K_{j}^{\mp 1} \quad, \quad \gamma\left(F_{j}\right)=-K_{j} F_{j}
$$

and a co-unit $\epsilon$

$$
\epsilon\left(E_{j}\right)=0 \quad, \quad \epsilon\left(K_{j}^{ \pm 1}\right)=1 \quad, \quad \epsilon\left(F_{j}\right)=0
$$

it is straightforward to verify that the above is indeed a Hopf algebra. A final task is to implement quasi-triangularity in the Hopf algebra, which is done by defining the universal $R$ matrix to be the braiding matrix of two screened vertex operators. We refer to [7] for details.

Let us finally summarize the results for the (anti-)commutation relations

$$
\begin{aligned}
K_{i} K_{j} & =K_{j} K_{i} \\
K_{i} E_{j} & =e^{i \pi \Omega_{i j}} E_{j} K_{i} \\
K_{i} F_{j} & =e^{-i \pi \Omega_{i j} F_{j} K_{i}} \\
E_{i} F_{j}-(-1)^{p\left(E_{i}\right) p\left(F_{j}\right)} F_{j} E_{i} & =\delta_{i j} \frac{K_{i}-K_{i}^{-1}}{q_{i}-q_{i}^{-1}}
\end{aligned}
$$

\section{3 q-deformed Enveloping Lie Superalgebras}

In this section we shall illustrate the above construction by considering the recently obtained explicit results on screening currents in affine current superalgebras [8]. Let us first review some of the results in that work while fixing our notation.

The rank of the underlying Lie superalgebra is $r$. Choose a set of simple roots $\left\{\alpha_{i}\right\}_{i=1, \ldots, r}$ such that the subset $\tau$ of $\{1, \ldots, r\}$ corresponds to the odd simple roots. The parities of the Chevalley generators are then defined by

$$
\begin{aligned}
p\left(H_{i}\right) & =0 \\
p\left(E_{i}\right)=p\left(F_{i}\right) & = \begin{cases}1, & i \in \tau \\
0, & i \notin \tau\end{cases}
\end{aligned}
$$

In general, the parity of a raising $E_{\alpha}$ or a lowering operator $F_{\alpha}$ is 0 (1) if the corresponding root $\alpha$ is even (odd). For the purpose of definiteness we choose a standard convention where the Cartan matrix is given by

$$
A_{i j}= \begin{cases}\frac{2 \alpha_{i} \cdot \alpha_{j}}{\alpha_{i}^{2}} & , \quad \alpha_{i}^{2} \neq 0 \\ \alpha_{i} \cdot \alpha_{j} & , \quad \alpha_{i}^{2}=0\end{cases}
$$


We shall determine the $\Omega$ matrices (2) working in the framework of free field realizations [16, 10, 11, 8]. The free field realization of an affine current superalgebra is based on a bosonic scalar field $\varphi_{i}$ for every Cartan index $i$ and a pair of free bosonic (fermionic) ghost fields $\left(\beta_{\alpha}, \gamma^{\alpha}\right)$ for every positive even (odd) root $\alpha$. Their defining operator product expansions are

$$
\begin{aligned}
\varphi_{i}(z) \varphi_{j}(w) & =G_{i j} \ln (z-w) \\
\beta_{\alpha}(z) \gamma^{\alpha^{\prime}}(w) & =\frac{\delta_{\alpha}^{\alpha^{\prime}}}{z-w}
\end{aligned}
$$

$G_{i j}$ is the Cartan part of the Cartan-Killing form. The affine current superalgebra is characterized by the level or central extension $k$ which may conveniently be renormalized using the dual Coxeter number $h^{\vee}$ by defining the parameter $t$ to be

$$
t=k+h^{\vee}
$$

Since we don't need the explicit free field realization of the affine currents we shall only review the result for the screening currents [8] which may be written in terms of the matrix $C$ defined by

$$
C_{a}^{b}(\gamma(w))=-\gamma^{\alpha}(w) f_{\alpha, a}{ }^{b}
$$

We shall use the convention of implicit summation over positive roots. $f_{a, b}{ }^{c}$ are the structure constants. In [8] (we refer to that work for more details) it is found that the screening currents are of the form

$$
S_{j}(w)=-:[B(-C(\gamma(w)))]_{\alpha_{j}}^{\alpha^{\prime}} \beta_{\alpha^{\prime}}(w):: e^{-\alpha_{j}(H \cdot \varphi(w)) / \sqrt{t}}:
$$

where

$$
\partial \varphi_{i}(z) \alpha(H \cdot \varphi(w))=\frac{\alpha\left(H_{i}\right)}{z-w}
$$

The function $B$ is the generating function for the Bernoulli numbers

$$
B(u)=\frac{u}{e^{u}-1}=\sum_{n \geq 0} \frac{B_{n}}{n !} u^{n}
$$

while the vertex operators are simply given by

$$
V_{\lambda}(z)=: e^{\lambda \cdot \varphi(z) / \sqrt{t}}:
$$

It is now straightforward to verify that the $\Omega$ matrices are given by

$$
\begin{aligned}
\Omega_{\lambda \lambda^{\prime}} & =\lambda \cdot \lambda^{\prime} / t \\
\Omega_{i \lambda} & =-\alpha_{i} \cdot \lambda / t \\
\Omega_{i j} & =\alpha_{i} \cdot \alpha_{j} / t
\end{aligned}
$$

After introducing the deformation parameter

$$
q=e^{i \pi / t}
$$


and the symmetrizing parameters $D_{i}$ (which symmetrize the Cartan matrix, $D_{i} A_{i j}$ is symmetric)

$$
D_{i}=\left\{\begin{array}{lll}
\alpha_{i}^{2} / 2 & , & \alpha_{i}^{2} \neq 0 \\
1 & , & \alpha_{i}^{2}=0
\end{array}\right.
$$

we choose the deformation parameters $q_{i}$ of the preceding section as

$$
q_{i}=q^{D_{i}}
$$

Using these and the results (31) for the $\Omega$ matrices, it is immediately found that the (anti-)commutator relations (21) may be written as

$$
\begin{aligned}
K_{i} K_{j} & =K_{j} K_{i} \\
K_{i} E_{j} & =q_{i}^{A_{i j}} E_{j} K_{i} \\
K_{i} F_{j} & =q_{i}^{-A_{i j}} F_{j} K_{i} \\
E_{i} F_{j}-(-1)^{p\left(E_{i}\right) p\left(F_{j}\right)} F_{j} E_{i} & =\delta_{i j} \frac{K_{i}-K_{i}^{-1}}{q_{i}-q_{i}^{-1}}
\end{aligned}
$$

Now the question arises as to what quantum superalgebra the above construction corresponds. Owing to its obvious resemblance, the natural proposal would be that it is the $q$-deformed universal enveloping algebra of the underlying Lie superalgebra $\mathbf{g}$, namely円 $\mathcal{U}_{e^{i \pi / t}}(\mathbf{g})$. However, as it has been observed by Scheunert 19, the problem of presenting Lie superalgebras (and thus their quantum deformations) by Serre type relations is not a trivial one. It occurs that one needs supplementary relations not naively defined by the Cartan matrix. We leave it for future investigations to determine what set of Serre type relations the above construction respects.

\section{Conclusion}

In this letter we have generalized the Gomez-Sierra construction of quantum groups to the case of supergroups and illustrated the generalization using recent results on screening currents in affine current superalgebra.

Another possible generalization is to include screening currents of the second kind. Based on steps already taken along those lines [10] we anticipate that in the purely bosonic case one should encounter a semi-direct sum of two $q$-deformed universal enveloping algebras of the underlying Lie algebra and of the transposed of that, respectively, with the two $q$ 's being related as $q^{\prime}=q^{t^{2}}$. However, as it has been demonstrated in [12], screening currents of the second kind are very complicated objects. We intend to come back elsewhere with more discussions on screening currents of the second kind and of the associated quantum groups.

\section{Acknowledgement}

The author is financially supported by the Danish Natural Science Research Council, contract no. 9700517.

\footnotetext{
${ }^{1}$ Admissible representations [17, 18] are characterized by the parameter $t$ being rational. This means that in those cases the construction corresponds to a $q$-deformation with $q$ a root of unity.
} 


\section{References}

[1] P. Kulish and E.K. Sklyanin, in Integrable Quantum Field Theories, Twarminne, 1981, J. Hietarinta and C. Montonen (Eds.), Lecture Notes in Physics 151 p. 61, (Springer, 1982);

L.D. Faddeev, in Recent Advances in Field Theory and Statistical Mechanics, Les Houches, Session XXXIX 1982, J.-B. Zuber and R. Stora (Eds.), p.561, (NorthHolland, 1984);

P. Kulish and N.Yu. Reshetikhin, J. Sov. Math. 23 (1983) 2435

[2] V.G. Drinfeld, Sov. Math. Dokl. 32 (1985) 254;

M. Jimbo, Lett. Math. Phys. 10 (1985) 63

[3] P. Kulish and N.Yu. Reshetikhin, Lett. Math. Phys. 18 (1989) 143

[4] M. Chaichian and P. Kulish, Quantum Superalgebras, q-Oscillators and Applications, in Johns Hopkins Workshop 1990, p.213, CERN-TH-5969-90

[5] C. Gomez and G. Sierra, Phys. Lett. B 240 (1990) 149;

C. Gomez and G. Sierra, Nucl. Phys. B 352 (1991) 791

[6] C. Ramirez, H. Ruegg and M. Ruiz-Altaba, Phys. Lett. B 247 (1990) 499;

C. Ramirez, H. Ruegg and M. Ruiz-Altaba, Nucl. Phys. B 364 (1991) 195

[7] C. Gomez and G. Sierra, in Proc. of the Trieste 1990 Summer School (World Scientific, 1991);

C. Gomez, M. Ruiz-Altaba and G. Sierra, Quantum Groups in Two-dimensional Physics (Cambridge Univ. Press, 1996)

[8] J. Rasmussen, Free Field Realizations of Affine Current Superalgebras, Screening Currents and Primary Fields, NBI-HE-97-15, hep-th/9706091, accepted for publication in Nucl. Phys. B

[9] M. Bershadsky and H. Ooguri, Commun. Math. Phys. 126 (1989) 49

[10] J. Rasmussen, Applications of Free Fields in 2D Current Algebra, Ph.D. thesis (The Niels Bohr Institute), hep-th/9610167

[11] J.L. Petersen, J. Rasmussen and M. Yu, Nucl. Phys. B 502 (1997) 649

[12] J. Rasmussen, Screening Currents in Affine Current Algebras, q-alg/9711017

[13] Vl.S. Dotsenko and V.A. Fateev, Nucl. Phys. B 240[FS12] (1984) 312;

Vl.S. Dotsenko and V.A. Fateev, Nucl. Phys. B 251[FS13] (1985) 691

[14] G. Felder, Nucl. Phys. B 317 (1989) 215 [Erratum: B 324 (1989) 548]

[15] J.L. Petersen, J. Rasmussen and M. Yu, Nucl. Phys. B 457 (1995) 309;

J.L. Petersen, J. Rasmussen and M. Yu, Nucl. Phys. B 457 (1995) 343;

J.L. Petersen, J. Rasmussen and M. Yu, Nucl. Phys. B 481 (1996) 577 
[16] M. Wakimoto, Commun. Math. Phys. 104 (1986) 60

[17] V.G. Kac and D.A. Kazhdan, Adv. Math. 34 (1979) 97

[18] V.G. Kac and M. Wakimoto, Proc. Natl. Acad. Sci. USA 85 (1988) 4956;

V.G. Kac, and D.A. Kazhdan, Adv. Ser. Math. Phys., Vol. 7 (World Scientific, 1989), p. 138

[19] M. Scheunert, Lett. Math. Phys. 24 (1992) 173 